JOURNAL OF SYNCHROTRON RADIATION

ISSN 1600-5775

Received 26 May 2020

Accepted 5 October 2020

Edited by P. A. Pianetta, SLAC National Accelerator Laboratory, USA

Keywords: X-ray reflectivity; mid-spatial frequency; surface waviness; X-ray optics.

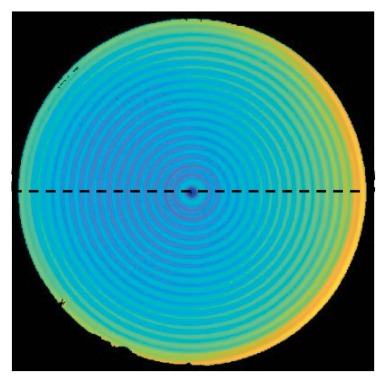

C 2021 International Union of Crystallography

\section{The effect of longer-range waviness on X-ray reflectivity measurements}

\author{
Jacob A. Cole, ${ }^{a *}$ Jefferson A. Cuadra, ${ }^{b}$ Robert M. Panas ${ }^{b}$ and Stuart T. Smith \\ aDepartment of Mechanical Engineering and Engineering Science, The University of North Carolina at Charlotte, \\ Charlotte, NC 28223, USA, and ${ }^{\mathbf{b}}$ Materials Engineering Division, Lawrence Livermore National Laboratory, \\ Livermore, CA 94551, USA. *Correspondence e-mail: jcole50@uncc.edu
}

A model for calculating the X-ray reflectivity (XRR) of surfaces to extract both roughness and waviness features is presented. Expressions of reflectivity intensity are derived as a function of root-mean-square (RMS) roughness $\sigma$, RMS waviness $\sigma_{L}$, and the cut-off frequency between the features $\omega_{0}$. Experiments were conducted at the Advanced Light Source at Lawrence Berkeley National Laboratory, beamline 8.3.2, on BK7 glass manufactured with a multi-step polishing process to validate the model, and were compared with atomic force microscopy (AFM), Fizeau interferometry and surface profilometry measurements. The parameter results and their deviations for XRR measurements were $\sigma=2.9 \pm 0.2 \mathrm{~nm}$ and $\sigma_{L}=14.6 \pm 0.5 \mathrm{~nm}$ with a wavelength cut-off of $1 /(18 \pm 2) \mu \mathrm{m}^{-1}$, while the results from the AFM, Fizeau and profilometry measurements were $\sigma_{\mathrm{AFM}}=3.4 \pm 0.4 \mathrm{~nm}, \sigma_{L \text {, Fizeau }}=21.6 \mathrm{~nm}, \sigma_{\text {prof }}=$ $4.0 \pm 0.1 \mathrm{~nm}$, and $\sigma_{L, \text { prof }}=21.4 \pm 0.1 \mathrm{~nm}$ with cut-offs for the profilometry and Fizeau measurements limited to frequencies of $(1 / 16) \mu \mathrm{m}^{-1}$ to $(1 / 4) \mathrm{mm}^{-1}$.

\section{Introduction}

The measurement of optical surface waviness is becoming more important because of its impact on the performance of many optics (Youngworth \& Stone, 2000; Tamkin et al., 2010). Mid-spatial frequency errors arise in the manufacturing process from deterministic grinding, polishing, or diamond turning and can leave grating-like patterns on a surface (Heinzel \& Grimme, 2006; Cheng et al., 2007). Today's highperformance optics span a broad range of technologies such as extreme ultraviolet (EUV) lithography (Bakshi et al., 2017), synchrotron optics and mirrors (Wiegmann et al., 2011; Wen et al., 2018), and freeform optics such as those used in defense, augmented (AR) and virtual reality (VR), and smartphone camera systems (Fang et al., 2013). To achieve the surface topography requirements of these optics a non-destructive tool providing process metrology such as the one presented here is of particular relevance.

Imperfections affecting optical performance are traditionally divided into form errors and micro-scale roughness with form errors typically being measured by optical and contact profilometers and roughness being measured by phase-shifting interferometry and atomic force microscopy (AFM). As requirements on the precision of such optics increase, so does the need to understand the effect of surface imperfections on optical performance which is typically expressed using the point spread function (PSF) (Raimondi \& Spiga, 2014) or, its Fourier equivalents, the optical transfer function (OTF) or modulation transfer function (MTF) (de Groot, 2019). Parks (2008) demonstrated that surface deviations in the region 
between form and roughness must be considered. This region of features is called waviness in the field of surface topography and mid-spatial frequencies in the field of optical surface analysis. Waviness causes a spread in the area of the PSF. Sometimes referred to as 'flare' in EUV lithography tools (Deck \& Evans, 2005), this 'ripple error' scatters light from bright regions of the image to dark regions, reducing contrast (Bjorkholm, 1998). Additionally, mid-spatial frequency errors are of considerable concern in the field of visible optics and, with many other error process related sources being effectively addressed, are becoming a major factor limiting optical performance (Parks, 2008; Tamkin, 2016; Hull et al., 2012). These mid-spatial frequency errors are typically considered to range from wavelengths around $80 \mu \mathrm{m}$ to $3 \mathrm{~mm}$ although selection of these limits will depend on particular industrial processes, optical wavelengths, and functional requirements. Early examples of X-ray reflectivity (XRR) photographic methods being applied to the measurement of long-range nanometre-level surface profiles from optical and semiconductor components are given by Aschenbach (1985), Lindsey et al. (1988) and Berujon \& Ziegler (2012).

$\mathrm{XRR}$ is one of the most sensitive methods for roughness measurements, even more sensitive than scanning tunneling microscopes (Lengeler \& Hüppauff, 1993). The large spatial wavelength cut-off for XRR is proportional to the X-ray beam size and the lower-wavelength cut-off is on the order of the X-ray wavelength $(\sim 1 \AA)$. This sensitivity to roughness and its ability to measure extremely small features makes XRR an increasingly important measurement method as the surface topography requirements on high-performance optics increase. Due to the sensitivity of XRR to roughness, many engineering surfaces with large roughness ( $\sigma>15 \mathrm{~nm}$ RMS) are difficult to measure as the surface diffuse scatter will be high, meaning that the range of amplitudes measurable with this technique is small and may only be suited for the inspection of highly processed surfaces. Additionally, surfaces with high slopes will be difficult to measure due to the oblique view of XRR. Typically these types of optics have peak-tovalley waviness amplitudes of less than $100 \mathrm{~nm}$ and very low slopes and are not an issue for many optical measurement tools including XRR.

XRR has been an established, non-destructive tool for evaluating the surface and interfaces of highly polished optics, semiconductor wafers, and multi-layered thin films since the 1980s (Stoev \& Sakurai, 1999). For a broad review, see Dalliant \& Gilbert (2009). At glancing angles, X-rays are totally reflected from solid surfaces (Compton, 1923). At angles larger than the critical angle, for ideally smooth surfaces the XRR falls approximately with $\theta^{-4}$. This rate of attenuation in reflectivity is extremely sensitive and increases with the micro-scale roughness of the reflecting surface and is used as a quality control metric in the optical and semiconductor industries. To determine quantitative surface topographical measures values it is necessary to compare the measured decay with theoretical models of the reflectivity near grazing incidence. Most theories utilize the Born approximation for modeling reflection from weakly inter- acting rough surfaces and, while this is valid for incidence angles greater than the critical angle, a perturbation approach called the distorted wave Born approximation (DWBA) is used to determine both specular and diffuse scatter throughout the region of grazing-incidence angles (Sinha et al., 1988). While further iterative perturbations of this approach have demonstrated increased accuracy (de Boer, 1995), the DWBA theory, as outlined by Sinha et al. (1988), remains a common approach for many models (Marszałek et al., 2001; Su et al., 2012; Penkov et al., 2020). An alternate form of this DWBA theory (Bahr et al., 1993) without limitations on correlation length, which is usually made, is used in this article; however, the extension presented in this work can be convolved with any of the above roughness models.

To measure the form, waviness and roughness of a surface, multiple measurement methods are required with each instrument needing to have an overlapping instrument transfer function with the one used before it. Typical measurement systems include contact or optical profilometers for form measurement, Fizeau or scanning white light interferometry (SWLI) for mid-spatial and large-scale roughness measurement, and AFM for high spatial frequency roughness measurement. On large optics such as the ones used in synchrotrons and EUV lithography tools (which can be up to hundreds of $\mathrm{mm}$ ), multiple SWLI images must be stitched together to measure the entire optic. This stitching leads to compounding errors from multiple sources (e.g. small slope errors in individual images that accumulate with the number of stitched images) that are difficult to quantify (Wiegmann $e t$ al., 2011). Furthermore, optical measuring machines and AFM are highly susceptible to environmental and vibration effects where lateral resolution is reduced (de Groot, 2019). In contrast, the small incident angles in XRR result in the illumination of a large lateral area of the surface, allowing for a single, laterally averaged measurement, of large optics. Additionally, as the overall reflectivity from the surface is being directly measured, uncoupled vibrations between the source, sample, and detector do not affect the measurement result.

The purpose of this work is twofold: to provide a tool to simultaneously measure nanometre-level surface roughness and large surface waviness characteristics of high-performance optics and mirrors, and to extend the range of surfaces measurable by XRR to include surfaces with distinct spatial surface components. To illustrate the unique topographic measurement space that can be occupied by XRR, Fig. 1 maps the capabilities of various surface measuring instruments onto a plot of measurable lateral spatial frequency content range typically necessary for surfaces of high-performance optics. The space occupied by XRR typically encompasses that similar to AFM but extends to sub-nanometre spatial wavelengths. The incorporation of waviness separation further extends XRR into the mid-spatial region as a single measurement process. As a consequence this new method for analyzing XRR measurements could eliminate the need to use multiple instruments when the waviness and roughness of an optic is desired such as when characterizing lithography tool 
components, X-ray telescope optics, or high-quality synchrotron mirrors (Derst et al., 1997).

\section{Formalism}

Modeling of specular and diffuse scatter in grazing-incidence $\mathrm{X}$-ray reflectivity with nanometre-level surface roughness can be considered relatively well understood (Parratt, 1954; Croce et al., 1972; Sinha et al., 1988; Bahr et al., 1993; Bowen \& Tanner, 1993; de Boer, 1995). To determine the effect of longer-range waviness, it is reasonable to consider a surface initially having a uniform spectral density, $S_{0}(\omega)$, for which the higher-frequency components are substantially attenuated using a low-pass filter. In terms of a manufacturing process this smoothing might correspond to a final fine finishing process where roughness values are reduced to a few nanometres or less. In this case the reduction of these high-frequency components will result in a shorter correlation length surface roughness that is considered in most reflectivity models; for a review, see Stoev \& Sakurai (1999). Typically, the longer-range features that remain from previous surface modification processes are considered to represent the waviness features of the final surface. Such a surface is modeled as two, superposed topographies, one containing relatively long correlation lengths and large amplitude; the other being the short spatial wavelengths representing the nanometre or sub-nanometre roughness of the final finishing process. Modeling the longwavelength features by a first-order filter with a cut-off at $\omega_{0}$, the spectral density $S_{\mathrm{f}}$ of the filtered surface profile is given by

$$
S_{\mathrm{f}}(\omega)=\frac{S_{0}(\omega)}{\left[1+\left(\omega / \omega_{0}\right)^{2}\right]} .
$$

Using the Weiner-Kintchine theorem, the auto-correlation function $R_{L}(\tau)$ corresponding to this spectral density is given by the inverse Fourier transform

$$
\begin{aligned}
R_{L}(\tau) & =2 \omega_{0}^{2} \int_{0}^{\infty} \frac{S_{0}}{\omega_{0}^{2}+\omega^{2}} \exp (j \omega \tau) \mathrm{d} \omega \\
& =2 \omega_{0}^{2} \int_{0}^{\infty} \frac{S_{0}}{\omega_{0}^{2}+\omega^{2}} \cos (\omega \tau) \mathrm{d} \omega \\
& = \begin{cases}S_{0} \pi \omega_{0} \exp \left(-\omega_{0} \tau\right) & \text { if } \tau>0, \\
S_{0} \pi \omega_{0} \exp \left(\omega_{0} \tau\right) & \text { if } \tau<0 .\end{cases}
\end{aligned}
$$

This can be written in the form

$$
R_{L}(\tau)=\sigma_{L}^{2} \exp \left(-\left|\omega_{0} \tau\right|\right), \quad \tau>0,
$$

where

$$
\sigma_{L}^{2}=S_{0} \pi \omega_{0}
$$

Hence $\omega_{0}$ can be considered as the correlation length of the filtered surface. From Longuet-Higgins (Longuet-Higgins, 1957), for small slopes, the probability density $p(\zeta)$ of the local slope $\zeta$ of a random surface is approximately Gaussian,

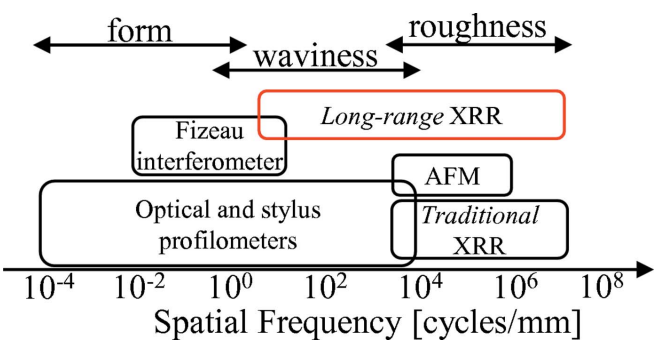

Figure 1

Plot of the measurable spatial frequency range of various surface measurement instruments. The extension of XRR presented in this work, 'Long-range' XRR, overlaps a substantial amount of the waviness and roughness regions and can be used instead of multiple measuring instruments.

$$
p(\zeta)=\frac{1}{\left(2 \pi m_{2}\right)^{1 / 2}} \exp \left(-\frac{\zeta^{2}}{2 m_{2}}\right),
$$

where $m_{2}$ is the mean square surface slope that can be computed from the integral of the second moment of the power spectral density (see Nayak, 1971; Newland, 1993). Hence

$$
\begin{aligned}
m_{2} & =\int \omega^{2} S_{f}(\omega) \mathrm{d} \omega \\
& =\frac{\sigma_{L}^{2} \omega_{0}}{\pi} \int \frac{\omega^{2}}{\omega_{0}^{2}+\omega^{2}} \mathrm{~d} \omega .
\end{aligned}
$$

This integral does not converge over all limits. However, it is reasonable to consider only the spatial frequencies longer than the correlation length corresponding to the X-ray scale surface roughness (or integer divisors thereof). Based on this assumption, the mean slope is given by

$$
\begin{aligned}
m_{2} & =\frac{2 \sigma_{L}^{2} \omega_{0}}{\pi} \int_{0}^{\omega_{0} / n} \frac{\omega^{2}}{\omega_{0}^{2}+\omega^{2}} \mathrm{~d} \omega \\
& =\frac{2 \sigma_{L}^{2} \omega_{0}}{\pi}\left[\frac{1}{n}-\tan ^{-1}\left(\frac{1}{n}\right)\right] .
\end{aligned}
$$

For $n=1$ the mean slope is given by

$$
\begin{aligned}
m_{2} & =\sigma_{L}^{2} \omega_{0}^{2}\left(\frac{4-\pi}{2 \pi}\right) \\
& =0.137 \sigma_{L}^{2} \omega_{0}^{2} .
\end{aligned}
$$

To determine the influence of these waviness features on $\mathrm{X}$-ray reflectivity, it is necessary to consider the distribution of slopes encountered by a beam incident at an angle $\theta$. For a flat surface with roughness $\sigma$ greater than about $3 \mathrm{~nm}$, first-order DWBA models break down and the specular reflectance $R(\theta)$ can be obtained analytically with a hyperbolic tangent interface function from (Bahr et al., 1993)

$$
R(\theta)=\frac{\sinh \left(\sigma\left\{\frac{\pi^{3} / 2}{2}\left[q_{z}(\theta)-q_{z, t}(\theta)\right]\right\}\right)}{\sinh \left(\sigma\left\{\frac{\pi^{3} / 2}{2}\left[q_{z}(\theta)+q_{z, t}(\theta)\right]\right\}\right)} G .
$$

For engineering surfaces with high polish, i.e. $\sigma<10 \mathrm{~nm}$, the phase factor $G \simeq 1$ (Hamilton \& Pynn, 1991) and can be omitted. In the above equation, $q_{z}$ and $q_{z, t}$ are the normal 


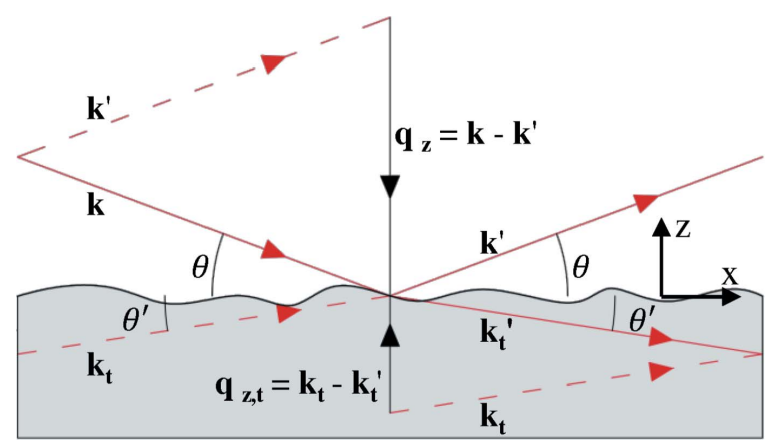

Figure 2

Diagram of the reflection and refraction of X-rays about an interface. $x$ is along the surface average, $z$ is normal to the surface, $\theta$ is the angle of incidence and angle of reflection, and $\theta^{\prime}$ is the angle of refraction.

components of the wavevector transfers in and out of the medium shown in Fig. 2 and are given by

$$
\begin{gathered}
q_{z}=\mathbf{k}-\mathbf{k}^{\prime}=2 k_{0} \sin \theta, \\
q_{z, t}=\mathbf{k}_{t}-\mathbf{k}_{t}^{\prime}=2 k_{0} \sin \theta^{\prime},
\end{gathered}
$$

where $\theta$ and $\theta^{\prime}$ are the incident and refracted angles of interaction by the $\mathrm{X}$-rays, related by

$$
\cos \theta^{\prime}=\frac{\cos \theta}{\cos \theta_{c}} .
$$

The critical grazing-incidence angle, $\theta_{c}=(2 \delta)^{1 / 2}$, is the angle below which the $\mathrm{X}$-rays experience total external reflection where $\delta$ is the refractive index unit decrement corresponding to the specific material and X-ray energy.

The intensity of the reflected X-rays can be determined from the square of the reflectance. To determine the effect of the waviness (or mid-spatial frequencies) on the measured intensity, it is necessary to determine the scattering by this mosaic of slopes that will result in an effective specular blurring over the range of surface slopes that can be considered to span $\pm 3 \sqrt{m_{2}}$. Consequently, for any incident angle $\theta$ the effect of the surface mosaic on the measured intensity can be determined from the integral

$$
\begin{aligned}
I(\theta) & =\int_{-3 \sqrt{m_{2}}}^{3 \sqrt{m_{2}}} R(\theta) * R(\theta) P(\zeta) \mathrm{d} \zeta \\
& =2 \int_{0}^{3 \sqrt{m_{2}}}|R(\theta+\zeta)|^{2} P(\zeta) \mathrm{d} \zeta
\end{aligned}
$$

Equation (13) contains both of the surface roughness and waviness terms and is used to fit the experimental data in Section 4. A comparison of equations (9) and (13) is presented in Fig. 3.

\section{Experimental}

Measurements were carried out at the Advanced Light Source at Lawrence Berkeley National Laboratory, beamline 8.3.2 (MacDowell et al., 2012). X-ray energies from 14-22 keV were selected with a monochromator. The beam was constrained with a $100 \mathrm{~m}$ tungsten pinhole and had a flat intensity distribution. The sample-to-detector distance was $250 \mathrm{~mm}$. The optical system consisted of a $50 \mathrm{~m}$ LuAG scintillator and $2 \times$ lenses with a PCO.Edge multipixel detector, resulting in a pixel size of $3.22 \mathrm{~m}$. For each reflectivity scan, 100 images were collected over $0.5^{\circ}$ of rotation with an angular step size of $0.005^{\circ}$ using up to $500 \mathrm{~ms}$ exposure time depending on the energy selected.

A reference image was first collected of the beam for the use of normalization. Starting with the incident beam parallel to, and half-blocked by, the sample face, the sample rotation was incremented and a single image was collected for each point on the reflectivity curve. From each image, the initial $\left(\theta=0^{\circ}\right)$ image was subtracted and the image was summed in the vertical direction. Non-negative intensity in the subtracted, vertically summed images was integrated to extract the total intensity of the reflected beam for each incident angle.

A BK7 optical flat was chosen for measurement. This sample had a nominal flatness of $\lambda / 4(\lambda=633 \mathrm{~nm})$ and a sinusoidal profile was polished into the planar surface using magnetorheological finishing (Shahinian, 2018). To test the robustness of the theory, a large amplitude (approximately $75 \mathrm{~nm}$ peak-to-valley) and relatively high frequency (approximately 0.5 cycles $\mathrm{mm}^{-1}$ ) waviness was polished into the surface, providing an extreme example of the type of surface that might be seen in high-performance optics. The reflecting surface was measured using AFM with a Dimension 3100 manufactured by Digital Instruments to investigate the high-frequency, low-amplitude roughness. Profile traces, performed with a Mahr MarSurf LD260 profilometer capable of $0.8 \mathrm{~nm}$ resolution, were taken along the beam footprint path to measure the higher-amplitude, lower-frequency waviness features. 2D areal measurements of the waviness were performed with a Zygo Verifire Fizeau interferometer with a 4 -inch field of view and RMS repeatability of $<0.06 \mathrm{~nm}$. Measurement results from the profilometer are presented in Fig. 6 and Fizeau interferometer results are presented in Fig. 5.

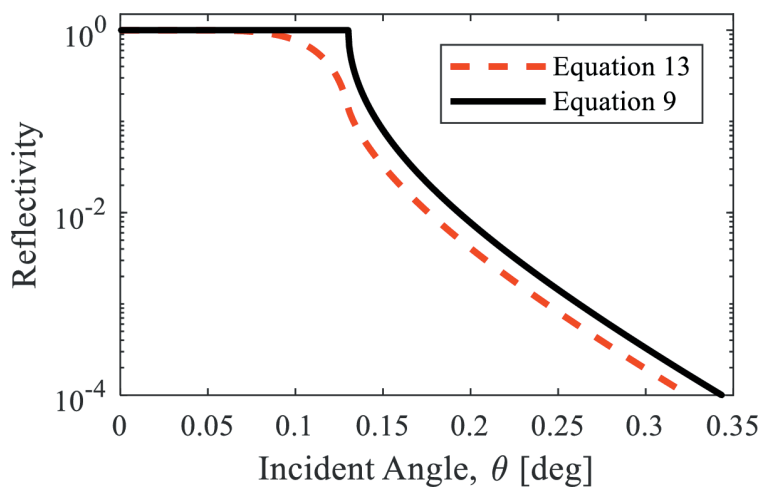

Figure 3

The computed reflectivity of Schott glass at $14 \mathrm{keV}$ using equations (9) and (13). Surface properties were $\sigma=2 \mathrm{~nm}, \sigma_{L}=20 \mathrm{~nm}$, and $\omega_{0}=1 / 15 \mu \mathrm{m}$. The waviness contributes to an overall decrease in reflectivity and a rounding-off at the critical angle. 
Table 1

Tabulated results from the XRR curve fits in Fig. 4.

\begin{tabular}{llll}
\hline & $\sigma(\mathrm{nm})$ & $\sigma_{L}(\mathrm{~nm})$ & $\omega_{0}\left(\mathrm{~m}^{-1}\right)$ \\
\hline $14 \mathrm{keV}$ & 2.8 & 14.2 & $1 / 17$ \\
$16 \mathrm{keV}$ & 3.1 & 14.0 & $1 / 20$ \\
$18 \mathrm{keV}$ & 2.8 & 15.3 & $1 / 20$ \\
$22 \mathrm{keV}$ & 3.1 & 14.8 & $1 / 15$ \\
\hline
\end{tabular}

\section{Results}

The extracted reflectivity information for various energies is shown in Fig. 4. All data were normalized to the reference image for each energy data set. A MATLAB program was used to iteratively search for best fit of the experimental data against equation (13). The fitting algorithm consisted of a Nelder-Mead simplex multi-dimensional unconstrained optimizer to minimize a squared cost function solving for RMS surface roughness $\sigma$, RMS surface waviness $\sigma_{L}$ and cut-off frequency $\omega_{0}$. Data obtained below $0.05^{\circ}$ do not lie on the curve fit due to the beam footprint being larger than the sample at these small incident angles. The resulting fit is presented with experimental results in Fig. 4.

The extrapolated parameters from the best fit for each of the four energies were averaged resulting in $\sigma=2.9 \pm 0.2 \mathrm{~nm}$, $\sigma_{L}=14.6 \pm 0.5 \mathrm{~nm}$ with a cut-off frequency between roughness and waviness of $\omega_{0}=1 /(18 \pm 2) \mu \mathrm{m}^{-1}$. Table 1 displays the results from the fitting algorithm for each energy. The sample was also subject to AFM, Fizeau interferometry (Fig. 5), and surface profilometry (Fig. 6) measurements. These measurements were bandwidth limited to extract both the surface roughness and waviness at the average frequency based on the XRR data fit results above.

When limited to feature frequencies of $(1 / 20) \mu \mathrm{m}^{-1}$ or greater, the AFM measurements resulted in an average roughness of $\sigma_{\mathrm{AFM}}=3.4 \pm 0.4 \mathrm{~nm}$. When limited to frequencies of $(1 / 16) \mu^{-1}$ to $(1 / 4) \mathrm{mm}^{-1}$, the Fizeau measurements resulted in a waviness of $\sigma_{L \text {, Fizeau }}=21.6 \mathrm{~nm}$. When limited to frequencies of $(1 / 16) \mu \mathrm{m}^{-1}$ to $(1 / 4) \mathrm{mm}^{-1}$, the profilometry measurements resulted in an average roughness of $\sigma_{\text {prof }}=$ $4.0 \pm 0.1 \mathrm{~nm}$ and a waviness value of $\sigma_{L \text {, prof }}=21.4 \pm 0.1 \mathrm{~nm}$.

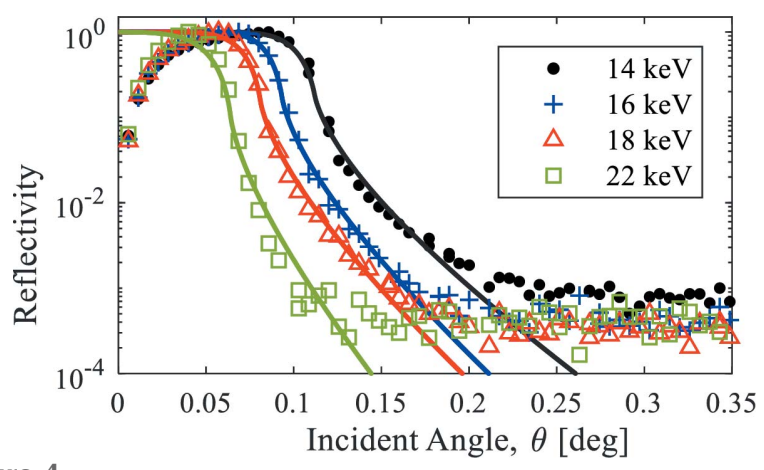

Figure 4

Experimental result of reflectivity for the measurement of a BK7 surface with roughness and waviness features for $E=14-22 \mathrm{keV}$. Theoretical fits to the data using equation (13) are shown as solid lines.

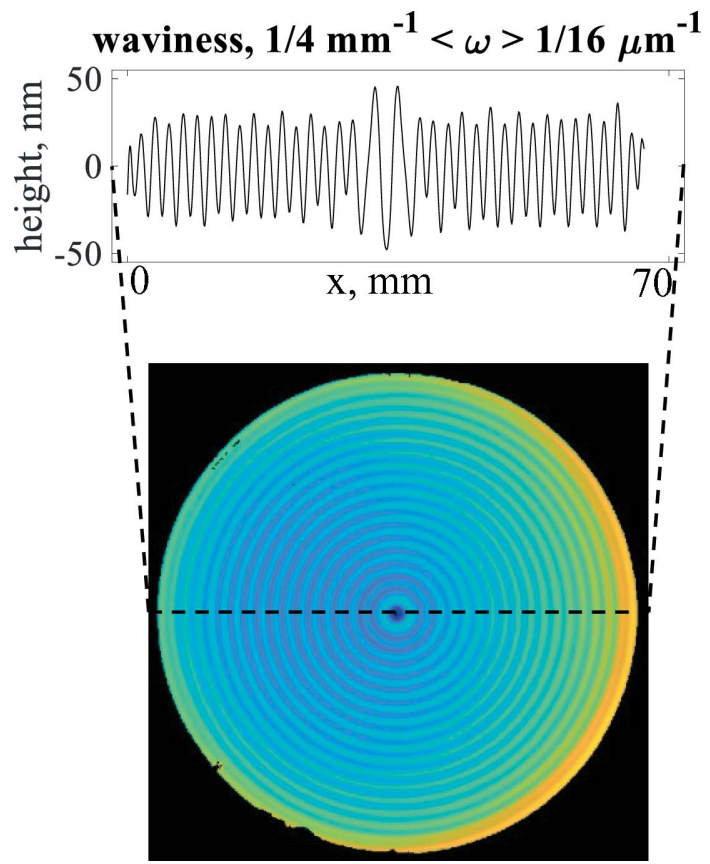

Figure 5

Result from the 2D areal Fizeau interferometer measurement of the BK7 flat. The highlighted trace is along the path that the XRR measurement was performed. The data were filtered at $(1 / 16) \mu \mathrm{m}^{-1}$; however, the Fizeau interferometer cannot measure frequencies in the higher roughness range and therefore the roughness plot contains no information (see Fig. 1).

A summary of the AFM and profilometry measurements is shown in Table 2.

Comparisons between XRR or AFM measurements are difficult due to the long, laterally averaged, measurement lengths of XRR. AFM measurements are typically acquired in a small region (i.e. $40 \mathrm{~m} \times 40 \mathrm{~m}$ in this experiment) while XRR measurement lengths are typically tens of millimetres long. Given a stationary, ergodic surface, the larger area averaging of the XRR measurement will result in lower
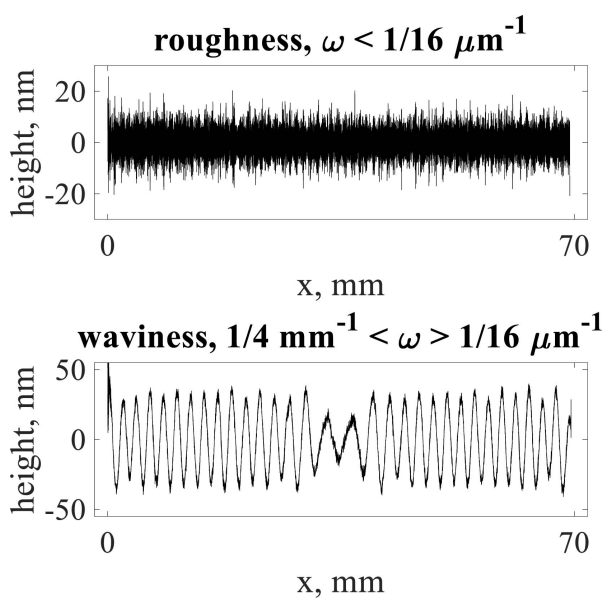

Figure 6

Filtered profilometer results, filtered at $(1 / 16) \mu \mathrm{m}^{-1}$. While the profilometer could easily measure the waviness features, the high-frequency content includes instrument noise. 
Table 2

Tabulated results from the AFM measurements and the profilometry measurements.

It is important to note that all XRR and profilometry measurements were taken along a single, longitudinal trace across the surface, while the AFM measurements are at five different $40 \mathrm{~m} \times 40 \mathrm{~m}$ patches along the same trace.

\begin{tabular}{llll}
\hline & $\sigma_{\mathrm{AFM}}(\mathrm{nm})$ & $\sigma_{\text {prof }}(\mathrm{nm})$ & $\sigma_{L, \text { prof }}(\mathrm{nm})$ \\
\hline Run 1 & 2.7 & 3.8 & 21.4 \\
Run 2 & 3.3 & 4.0 & 21.5 \\
Run 3 & 4.0 & 4.0 & 21.2 \\
Run 4 & 3.5 & 4.1 & 21.6 \\
Run 5 & 3.4 & 4.1 & 21.5 \\
\hline
\end{tabular}

variance than the AFM measurement. Profilometry measurements use a tactile probe with a finite radius, typically around $100 \mathrm{~nm}$ or larger and considerably larger than AFM tip radii. This stylus probe tip size will apply a low-pass filter to the measurement, whereas XRR measurements, due to the short wavelengths of X-rays, can probe surface features having significantly higher spatial frequencies than AFM and profilometry. Optical surface measuring instruments such as Fizeau interferometers have much smaller lateral resolution when compared with AFM or XRR and are typically only used for mid-spatial frequency and form measurements. In this case, the profilometry and Fizeau bandwidth limited waviness values agreed within about $1 \%$. On the other hand, spurious scattering and absorption of higher slopes on the surface will impact the reflectivity and influence the waviness result which could explain differences seen here and must be investigated in further studies. Additionally, the roughness values from X-ray reflectivity are sensitive to the reflectivity intensity being artificially high due to unfiltered diffuse scatter reaching the detector. This will cause the measurement result to be smoother and may contribute to variations seen in this work. Additions of slits or longer sample-to-detector distance may reduce the collected diffuse scatter. The model presented here assumes both the roughness and waviness are approximately Gaussian whereas some processed surfaces may have a height distribution that is non-Gaussian. This is a known disadvantage with XRR due to the dependence of the extracted parameters on the theoretical model of the surface (Mironov et al., 2008). As mentioned in the Introduction, many models for simulating X-ray reflectivity exist with different interface probability density functions: Gaussian (Croce et al., 1972), tanh (Bahr et al., 1993), $\cos ^{-2}$ (Lekner, 1987). It is important to use the correct model to approximate the reflectivity of the surface; however, surfaces subject to many fine finishing steps such as mechanical, ion beam, elastic emission, and magnetorheological polishing will approach Gaussian height distributions.

Upper limits on roughness amplitude typically are related to the radiation wavelength (Sinha et al., 1988), and the application of this method with soft X-ray sources could extend the range of amplitudes measurable but will increase the low spatial wavelength cut-off. Further evaluation of more complex surfaces is required to set formal limits on this technique.

\section{Conclusion}

A large number of engineering surface generation processes produce surfaces containing both longer-range waviness features and short-wavelength roughness features. This paper presents an extension of the currently established X-ray reflectivity theory to include surfaces having these two distinct surface components. The model combines the effect of a distribution of surface slopes caused by waviness with well established surface roughness models. The purpose of this model is not to replace existing XRR models but to provide a framework that expands the current theory to include surfaces having varying slopes due to mid-spatial frequency errors. This extension allows simultaneous measurement of highfrequency, low-amplitude roughness in addition to lowerfrequency, high-amplitude waviness features found on various engineering surfaces that have been produced using multiple reductive finishing processes.

\section{Acknowledgements}

The experimental work was performed under the auspices of the US Department of Energy by Lawrence Livermore National Laboratory under Contract DE-AC52-07NA27344 (LLNL-JRNL-805321). The authors would also like to acknowledge Advanced Light Source, which is supported by the Director, Office of Science, Office of Basic Energy Sciences, of the US Department of Energy under Contract No. DEAC02-05CH11231. The authors acknowledge Drs Brigid A. Mullany and Hossein Shahinian for supplying BK7 specimens.

\section{Funding information}

Funding for this research was provided by: Lawrence Livermore National Laboratory (contract No. DE-AC5207NA27344).

\section{References}

Aschenbach, B. (1985). Rep. Prog. Phys. 48, 579-629.

Bahr, D., Press, W., Jebasinski, R. \& Mantl, S. (1993). Phys. Rev. B, 47, 4385-4393.

Bakshi, V., Mizoguchi, H., Liang, T., Grenville, A. \& Benschop, J. (2017). J. Micro/Nanolithography MEMS MOEMS, 16, 1.

Berujon, S. \& Ziegler, E. (2012). Opt. Lett. 37, 4464-4466.

Bjorkholm, J. E. (1998). Intel Technol. J. Q3'98.

Boer, D. K. G. de (1995). Phys. Rev. B, 51, 5297-5305.

Bowen, D. K. \& Tanner, B. K. (1993). Nanotechnology, 4, 175-182.

Cheng, M., Cheung, C. F., Lee, W. B. \& To, S. (2007). Key Eng. Mater. 339, 400-406.

Compton, A. H. (1923). London Edinb. Dubl. Philos. Mag. J. Sci. 45, 1121-1131.

Croce, P., Névot, L. \& Pardo, B. (1972). Nouv. Rev. d'Opt. Appl. 3, $37-50$.

Dalliant, J. \& Gilbert, A. (2009). X-ray and Neutron Reflectivity: Principles and Applications. Springer.

Deck, L. L. \& Evans, C. (2005). Proc. SPIE, 5921, 73-80.

Derst, G., Handschuh, H., Schmidt, M. \& Werner, K. (1997). Proc. SPIE, 3152, 51-58.

Fang, F. Z., Zhang, X. D., Weckenmann, A., Zhang, G. X. \& Evans, C. (2013). CIRP Annals, 62, 823-846.

Groot, P. J. de (2019). Rep. Prog. Phys. 82, 056101. 
Hamilton, W. A. \& Pynn, R. (1991). Physica B, 173, 71-73.

Heinzel, C. \& Grimme, D. (2006). CIRP Ann. Manuf. Technol. 55, 581-584.

Hull, T., Riso, M. J., Barentine, J. M. \& Magruder, A. (2012). Proc. SPIE, 8353, 835329.

Lekner, J. (1987). Theory of Reflection of Electromagnetic and Particle Wavees. Developments in Electromagnetic Theory and Application. Martinus Nijhoff.

Lengeler, B. \& Hüppauff, M. (1993). Fresenius J. Anal. Chem. 346, 155-161.

Lindsey, K., Smith, S. T. \& Robbie, C. J. (1988). Annals CIRP, 37, 519522.

Longuet-Higgins, M. S. (1957). Philos. Trans. R. Soc. 249, 321-387.

MacDowell, A., Parkinson, D., Haboub, A., Schaible, E., Nasiatka, J. R., Yee, C. A., Jameson, J. R., Ajo-Franklin, J. B., Brodersen, C. R. \& McElrone, A. J. (2012). Proc. SPIE, 8506, 850618.

Marszałek, M., Jaworski, J., Michalik, A., Prokop, J., Stachura, Z., Voznyi, V., Bölling, O. \& Sulkio-Cleff, B. (2001). J. Magn. Magn. Mater. 226-230, 1735-1737.

Mironov, V. L., Udalov, O. G., Gribkov, B. A. \& Fraerman, A. A. (2008). J. Appl. Phys. 104, 064301.

Nayak, P. R. (1971). Trans. ASME: J. Lubr. Technol. 93, 398-407.

Newland, D. E. (1993). Random Vibrations, Spectral and Wavelet Analysis, 3rd ed. Longman Scientific.
Parks, R. E. (2008). Proc. SPIE, 7071, 46-54.

Parratt, L. (1954). Phys. Rev. 95, 359-369.

Penkov, O. V., Kopylets, I. A., Khadem, M. \& Qin, T. (2020). SoftwareX, 12, 100528.

Raimondi, L. \& Spiga, L. (2014). Astron. Astrophys. 573, A22.

Shahinian, H. (2018). Fiber Based Tools for Polishing Optical Materials. PhD thesis, University of North Carolina at Charlotte, USA.

Sinha, S., Sirota, E., Garoff, S. \& Stanley, H. (1988). Phys. Rev. B, 38, 2297-2311.

Stoev, K. S. \& Sakurai, K. (1999). At. Spectrosc. 54, 41-82.

Su, H. C., Lee, C. H., Lin, M. Z. \& Huang, T. W. (2012). Chin. J. Phys. 50, 291-300.

Tamkin, J. M. (2016). A study of image artifacts caused by structured mid-spatial frequency fabrication errors on optical surfaces. $\mathrm{PhD}$ thesis, University of Arizona, USA.

Tamkin, J. M., Milster, T. D. \& Dallas, W. (2010). Appl. Opt. 49, 48254835.

Wen, M., Kozhevnikov, I. V., Siewert, F., Buzmakov, A. V., Xie, C., Huang, Q., Wang, Z., Samoylova, L. \& Sinn, H. (2018). Opt. Express, 26, 21003.

Wiegmann, A., Stavridis, M., Walzel, M., Siewert, F., Zeschke, T., Schulz, M. \& Elster, C. (2011). Precis. Eng. 35, 183-190.

Youngworth, R. N. \& Stone, B. D. (2000). Appl. Opt. 39, 2198. 